\title{
From Identity to Militancy: The Shī'a of Hezbollah
}

\author{
Steven Childs
}

Due to rights restrictions,

this chapter is not available

in the digital edition of the book. 